\title{
EFFECT OF LIFESTYLE MODIFICATION IN TYPE II DIABETES MELLITUS INDIVIDUALS
}

\author{
AVANI BHOSALE ${ }^{1 *}$, POOVISHNU DEVI THANGAVELU²
}

${ }^{1}$ Department of Cardio Respiratory Sciences, Faculty of Physiotherapy, Krishna University, Karad, Maharashtra, India. ${ }^{2}$ Department of Cardiorespiratory Sciences, Faculty of Physiotherapy, Krishna University, Karad, Maharashtra, India. Email: avanibhosale@yahoo.com

Received: 10 September 2018, Revised and Accepted: 01 November 2018

ABSTRACT

Objectives: The objectives of this study were as follows: (1) To find the effect of conventional physiotherapy along with medications on Type II diabetes mellitus (DM) individuals and (2) to find the effect of lifestyle modification (LSM) and conventional physiotherapy with medications on Type II DM individuals.

Methods: Ethical clearance was obtained from the institutional ethical committee. A total of 70 Type II DM individuals were assessed and 64 were included in this study based on inclusion criteria, the individuals were allocated into two groups: Group A (n=32) received conventional physiotherapy along with prescribed diabetic medications and Group B $(n=32)$ received LSM and conventional physiotherapy with prescribed diabetic medications. Pre- and post-test were done for plasma glucose, serum insulin, glycated hemoglobin (HbA1c), total cholesterol, triglycerides, high-density lipoprotein-C, low-density lipoprotein by blood test, exercise tolerance by 6-min walk test, and quality of life (QOL) by SF-36 questionnaire, and the outcome measures were analyzed after 3 months.

Results: Intergroup statistical analysis for HbA1c ( $<<0.0001)$ and QOL ( $<<0.0001)$ revealed extremely significant difference post-intervention. Analysis of HbA1c test for Group A ( $\mathrm{p}=0.0652)$ was not significant and for Group B ( $<<0.0001)$ was extremely significant. Moreover, QOL was significant for Group A ( $\mathrm{p}=0.0004)$ as well as for Group B $(\mathrm{p}<0.0001)$.

Conclusion: The study results concluded that LSM was significantly effective in reducing HbA1c levels, exercise tolerance, plasma glucose, serum glycerine, and total cholesterol and improving QOL compared to conventional exercise.

Keywords: Lifestyle modification, Type II diabetes mellitus, Resistance exercises, Walking.

(C) 2019 The Authors. Published by Innovare Academic Sciences Pvt Ltd. This is an open access article under the CC BY license (http://creativecommons. org/licenses/by/4. 0/) DOI: http://dx.doi.org/10.22159/ajpcr.2019.v12i2.29668

\section{INTRODUCTION}

Type II diabetes mellitus (DM) is a long-term metabolic disorder that is characterized by high blood sugar, insulin resistance, and relative lack of insulin [1]. With the onset of this chronic condition and associated comorbidities, a life-long reduction in quality of life (QOL) and premature mortality due to micro- and macro-vascular complications occurs [2]. According to recent estimates, approximately $6.6 \%$ of people worldwide are having DM; in India, there are about 50.8 million adults with DM [3]. In Maharashtra, there are $9.3 \%$ of people with type II DM, $49 \%$ of males and $51 \%$ of females. Type II DM results from the body's inability to respond properly to the action of insulin produced by the pancreas. Type II DM is seen more commonly than type I DM [4]. The adverse effects of diabetic drugs such as sulfonylureas cause hypoglycemia or non-specific side effects (nausea, vomiting, constipation, headache, weight gain, and diarrhea) and metformin cause lactic acidosis or B12 deficiency [5]. As per previous studies, the cost of therapy is higher for DM. The percentage of expenditure was higher in low-income group and burden of therapy was directly proportional to the number of tablets, poor education, and occupational status and inversely proportional to income [6].

Lifestyle modification (LSM) involves altering long-term habits, typically of eating or physical activity, and maintaining the new behavior for months or years and can be used in all non-communicable diseases. LSM leads to moderate weight loss and increases activity levels $[7,8]$. In the long term, LSM prevents further complications and delays the complications of diabetes [9].

Physician assessed the individual. Necessary investigations were done. Then, they prescribe medications if needed. Exercise therapist assessed the individual and structured an exercise plan and observed the individual to make sure the individual is doing it with proper technique. She explained them about the importance of physical activity. Nutritionist assessed the individual and prepared a proper diet chart considering the blood glucose level and age of the subject. Behavior therapist assessed the individual. They observed the psychological state of the individual regarding their DM. They explained the individual about the medication schedule. The normal value of fasting glucose level (4.0-5.4 mol/d $)$ and $2 \mathrm{~h}$ after eating $(7.8 \mathrm{mmol} / \mathrm{dl})$. If the glucose value increases, then the individual should seek medical help immediately.

\section{METHODS}

Ethical clearance was obtained from the Institutional Ethical Committee, KIMSDU, Karad. The study included 70 individuals with Type II DM and divided into two groups. Exclusion criteria included currently undergoing other exercise programs, musculoskeletal disorders which disturb the balance and coordination, neurological or psychological disorder, diabetic foot, impaired sensation and infection, and blurred vision. 70 individuals with Type II DM were assessed, of which three were not meeting inclusion and exclusion criteria and three were not willing to participate in the study. 64 individuals were included in the study. After baseline assessment, which included blood test, glycated hemoglobin (HbA1c) test, and diabetic questionnaire (SF-36), they were allocated to respective groups using random sampling method. The first group (Group A $\mathrm{n}=32$ ) received advice for walking for $45 \mathrm{~min} /$ day for 5 days/week along with diabetic medications. The second group (Group $\mathrm{B} n=32$ ) received LSM and walking with diabetic medications.

\section{Sampling method}

a. Group A received advice for walking along with diabetic medications. 
Structured exercise program for Group B

a. Exercises for upper body: Push-ups, dips, planks, and biceps curls. Lower body exercises: Squatting, standing calf raise, leg lift, and prone hip extension. Trunk exercises: Pelvic bridging and prone extension. All exercises were done with $10-30$ s hold and 10 repetitions.

b. Diet that involves enough amounts of protein, limited intake of processed sugars and carbohydrates, protein and fat do not directly impact on glucose level but can be consumed in moderate amount to keep calories low.

c. Behavioral therapist explained those regarding safe eating habits, importance of physical therapy, avoiding risk factors, and avoiding their addictions.

d. Nurse explained the individual about the medication schedule. The normal value of fasting glucose level $(4.0-5.4 \mathrm{~mol} / \mathrm{dl})$ and $2 \mathrm{~h}$ after eating $(7.8 \mathrm{mmol} / \mathrm{dl})$. If the glucose value increases, then the individual should seek medical help immediately.

Group A enrolled 32 individuals, of which one individual dropped out of the study due to irregular follow-up and one individual due to transport issues. Group B included 32 individuals, of which one individual dropped out due to irregular follow-up and one individual due to some other personal reason. At the end of the study, a total of 60 individuals were taken for analysis.

\section{RESULTS}

Primary outcomes used for the result were plasma glucose levels, serum insulin levels, cholesterol, triglycerides, high-density lipoprotein (HDL)-C, low-density lipoprotein (LDL)-C, 6-min walk test, HbA1c, and SF-36 QOL questionnaire. The American Diabetes Association has recommended $\mathrm{HbA1c}$ as substitute for fasting blood glucose for diagnosis of diabetes. HbA1c is an indicator of long term as well as preceding 2-3 months (Tables 1-5) [10].

\section{Age distribution}

Group A involved total 30 individuals of which 8 individuals were of age 40-45, 8 individuals of age 45-50 and 14 individuals of age 50-55. Group B involved total of 30 individuals of which 15 individuals were of age 40-45, 5 individuals of age 45-50 and 10 individuals of age 50-55.

\section{Gender distribution}

Group A involved 30 individuals of which 11 were females and 19 were males. Group B involved 30 individuals of which 19 were females and 11 were males.

\section{Blood test}

Group A had fasting glucose level of 6.5, 2 hour glucose was 10.58 , fasting insulin was $11.22,2$ hour insulin was 51 , HbA1c was 6 , total cholesterol was 5.41, triglycerides was 2.11, (HDL)-C was 0.99 and (LDL)-C was 4.07. Group B had fasting glucose level of $6.29,2$ hour glucose was 8.73, fasting insulin was 7.47, 2 hour insulin was 29.3 , HbA1c was 6.2 , total cholesterol was 4.57 , triglycerides was 1.35 , (HDL)-C was 1.02 and LDL-C was 3.45 .

\section{6 min walk test}

6 minutes walk test result for group A was 528 and for group B was 598 .

\section{QOL questionnaire}

Within the group, the data were analyzed by paired t-test. Between the groups, the data were analyzed by unpaired t-test. $p$ and $t$ values were calculated.

\section{DISCUSSION}

Type II DM damages arteries and makes them target for hardening called atherosclerosis resulting in high blood pressure if not treated can lead to heart attack, stroke, and kidney failure [11]. The results of the study on LSM in Type II DM individuals concentrated on medications, diet, psychological aspect, and both aerobic and resistance exercise, and health education showed significant
Table 1: Age distribution

\begin{tabular}{llll}
\hline Age & Group A & Group B & Total \\
\hline $40-45$ & 8 & 15 & 23 \\
$45-50$ & 8 & 5 & 13 \\
$50-55$ & 14 & 10 & 24 \\
Total & 30 & 30 & 60 \\
\hline
\end{tabular}

Table 2: Gender distribution

\begin{tabular}{llll}
\hline Gender & Group A & Group B & Total \\
\hline Female & 11 & 19 & 30 \\
Male & 19 & 11 & 30 \\
Total & 30 & 30 & 60 \\
\hline
\end{tabular}

Table 3: Comparison between post-values of blood test in between Group A and Group B

\begin{tabular}{lll}
\hline Outcome measures & Group A & Group B \\
\hline Fasting glucose & 6.5 & 6.29 \\
2 h glucose & 10.58 & 8.73 \\
Fasting insulin & 11.22 & 7.47 \\
2 h insulin & 51 & 29.3 \\
HbA1c & 6 & 6.20 \\
Total cholesterol & 5.41 & 4.57 \\
Triglycerides & 2.11 & 1.35 \\
HDL-C & 0.99 & 1.02 \\
LDL-C & 4.07 & 3.45 \\
\hline
\end{tabular}

HDL: High-density lipoprotein-cholesterol, LDL: Low-density lipoproteincholesterol, HbA1c: Glycated hemoglobin

Table 4: This graph shows the difference in 6 min walk test score between Group A and Group B post-intervention

\begin{tabular}{lll}
\hline Outcome measure & Group A & Group B \\
\hline 6 min walk test & 528 & 598 \\
\hline
\end{tabular}

Table 5: The change in QOL in comparison between Group A and Group B post-intervention

\begin{tabular}{lll}
\hline QOL & Group A & Group B \\
\hline Physical function & 47.52 & 69.85 \\
Physical role limitation & 36.71 & 67.85 \\
Body pain & 60 & 88.92 \\
General health & 42.09 & 48.56 \\
Vital force & 53.9 & 70.14 \\
Social function & 59.14 & 88.92 \\
Psychological role limitation & 28.27 & 45 \\
Mental health & 63.13 & 77.37 \\
\hline
\end{tabular}

QOL: Quality of life

improvement on glycemic control, lipid profile, walking ability, and QOL in Type II DM individuals.

Resting and $2 \mathrm{~h}$ post-blood glucose concentrations were reduced in the exercise group, the glucose reduction is correlated with insulin sensitivity. Henrickson et al. stated that augmentation of insulinstimulated glucose transport in the skeletal muscle would be the possible mechanism. The results suggest that blood glucose and insulin sensitivity suggest people $>50$ years and long-standing diabetes individuals can improve glycemic control with LSM.

Kilpatrick et al. advocated that $\mathrm{HbA1c}$ represents the average glycemia control over the preceding 6-8 weeks [11]. This long-term variable with LSM shows reduction in Type II DM individuals in the earlier 
studies on LSM in Type II DM individuals, Sanghani et al. proved that HbA1c declines of $0.59 \%(p=0.030)$, the improvement in the present study with statistical significance must be due to physiological complex of HbA1c and larger sample size [2].

Dyslipidemia is common among people with Type II DM individuals. Dunstan et al. stated that with LSM, total cholesterol, triglycerides, and LDL are reduced, HDL is increased [11]. In the present study in Group B, total cholesterol $(\mathrm{p}<0.0001)$ is significantly reduced, triglyceride reduced with $\mathrm{p}<0.0001$ showing statistically significant reduction and HDL shows significant reduction.

Park et al. said that patients with Type II DM show lower muscle strength and skeletal mass compared to non-diabetic people. The reduction in muscle function results in insulin resistance [11]. LSM including aerobic exercise improves the muscle strength where overall results of 6 min walking program in Group B p<0.0001 and control group $\mathrm{p}=0.0006$. QOL in type II diabetes includes physical function, physical role limitation, body pain, general health, vital force, social function, psychological role limitation, and mental health.

\section{CONCLUSION}

Hence, on the basis of the result of our study, it was concluded that LSM was significantly effective in reducing HbA1c levels and improving QOL compared to aerobic exercise.

\section{ACKNOWLEDGMENT}

We would like to acknowledge the guidance and support of Ankita Mahipati Patil and Pratibha Pandekar from faculty of physiotherapy.

\section{AUTHORS' CONTRIBUTIONS}

Avani Dattaji Bhosale conducted literature review for this manuscript, developed introduction section of the manuscript together with the discussion of the study findings, collected data, and analyzed the data. T. Poovishnu Devi provided the description of the background information and participated in preparation of the manuscript.

\section{CONFLICTS OF INTEREST}

The author declares that there are no conflicts of interest concerning the content of the present study.

\section{REFERENCES}

1. Ramachandran A, Snehalatha C. Diabetes mellitus; epidemiology and basic considerations of diabetes. In: API Textbook of Medicine. $10^{\text {th }}$ ed., Vol. 1. New Delhi: Jaypee Brother's Medical Publishers; 2015. p. $467-4$

2. Sanghani NB, Parchwani DN, Palandurkar KM, Shah AM, Dhanani JV. Impact of lifestyle modification on glycemic control in patients with type 2 diabetes mellitus. Indian J Endocrinol Metab 2013;17:1030-9.

3. Harris MI, Hadden WC, Knowler WC, Bennett PH. Prevalence of diabetes and impaired glucose tolerance and plasma glucose levels in U.S. population aged 20-74 yr. Diabetes 1987;36:523-34

4. Patil RS, Gothankar SJ. Prevalence of type-2 diabetes mellitus and associated risk factors in an urban slum of Pune city, India. Natl J Med Res 2013;3:346-9.

5. Tripathi KD. Insulin, oral hypoglycaemic drugs and glucagon. In: Essentials of Medical Pharmacology. $6^{\text {th }}$ ed. New Delhi: Jaypee Brothers Medical Publishers; 2010. p. 258-81.

6. Kalpana M, Ruckmani A, Janti SS, Eerike M, Prabhu RL. Burden of therapy in patients suffering from diabetes mellitus and hypertension. Int J Pharm Pharm Sci 2015:9:210-5.

7. Foreyt JP, Poston WS. The challenge of diet, exercise and lifestyle modification in the management of the obese diabetic patient. Int $\mathrm{J}$ Obes Relat Metab Disord 1999;23 Supp1 7:S5-11.

8. Thent ZC, Das S, Henry LJ. Role of exercise in the management of diabetes mellitus: The global scenario. PLoS One 2013;8:e80436.

9. Patil VR, Thangvelu PD. Effectiveness of lifestyle modification on weight loss and quality of life in obese women with polycystic ovarian syndrome. Asian J Pharm Clin Res 2018;11:333-6.

10. Sherwani SI, Khan HA, Ekhzaimy A, Masood A, Sakharkar MK. Significance of hbAlc test in diagnosis and prognosis of diabetic patients. Biomark Insights 2016;11:95-104.

11. Tan S, Li W, Wang J. Effects of six months of combined aerobic and resistance training for elderly patients with a long history of type 2 diabetes. J Sports Sci Med 2012;11:495-501. 